OPEN ACCESS

Edited by:

Vitor Engracia Valenti,

São Paulo State University, Brazil

Reviewed by:

Andreas Richard Schwerdtfeger,

University of Graz, Austria

Phyllis Kravet Stein,

Washington University in St. Louis,

United States

*Correspondence:

Stephen B. R. E. Brown

stephen.brown@rdc.ab.ca

${ }^{\dagger}$ These authors have contributed equally to this work

Specialty section:

This article was submitted to

Autonomic Neuroscience,

a section of the journal

Frontiers in Neuroscience

Received: 20 May 2020 Accepted: 30 September 2020

Published: 22 October 2020

Citation:

Brown SBRE, Brosschot JF, Versluis A, Thayer JF and Verkuil $B$ (2020) Assessing New Methods

to Optimally Detect Episodes

of Non-metabolic Heart Rate

Variability Reduction as an Indicator of Psychological Stress in Everyday Life: A Thorough Evaluation of Six

Methods.

Front. Neurosci. 14:564123. doi: 10.3389/fnins.2020.564123

\section{Assessing New Methods to Optimally Detect Episodes of Non-metabolic Heart Rate Variability Reduction as an Indicator of Psychological Stress in Everyday Life: A Thorough Evaluation of Six Methods}

\author{
Stephen B. R. E. Brown ${ }^{1,2,3 * t}$, Jos F. Brosschot ${ }^{1,4 t}$, Anke Versluis ${ }^{1,5 t}$, Julian F. Thayer ${ }^{6 t}$ \\ and Bart Verkuil2,4t \\ 1 Department of Health, Medical, and Neuropsychology, Leiden University, Leiden, Netherlands, ${ }^{2}$ Leiden Institute for Brain \\ and Cognition, Leiden, Netherlands, ${ }^{3}$ Humanities and Social Sciences, Department of Psychology, Red Deer College, Red \\ Deer, AB, Canada, ${ }^{4}$ Department of Clinical Psychology, Leiden University, Leiden, Netherlands, ${ }^{5}$ Department of Public \\ Health and Primary Care, Leiden University Medical Centre, Leiden, Netherlands, ${ }^{6}$ Department of Psychological Science, \\ University of California, Irvine, Irvine, CA, United States
}

Frequent or chronic reduction in heart rate variability $(\mathrm{HRV})$ is a powerful predictor of cardiovascular disease, and psychological stress has been suggested to be a codeterminant of this reduction. Recently, we evaluated various methods to measure additional HRV reduction in everyday life and to relate these reductions to psychological stress. In the current paper, we thoroughly evaluate these methods and add two new methods in both newly acquired and reanalyzed datasets. All of these methods use a subset of $24 \mathrm{~h}$ worth of HRV and movement data to do so: either the first $10 \mathrm{~min}$ of every hour, the full $24 \mathrm{~h}$, a combination of $10 \mathrm{~min}$ from three consecutive hours, a classification of level of movement, the data from day $n$ to detect episodes in day $n$ +1 , or a range of activities during lab calibration. The method that used the full $24 \mathrm{~h}$ worth of data detected the largest percentage of episodes of reduced additional HRV that matched with self-reported stress levels, making this method the most promising, while using the first 10 min from three consecutive hours was a good runner-up.

Keywords: additional HRV, psychological stress, worry, cardiovascular disease, heart rate variability

\section{INTRODUCTION}

With cardiovascular disease being the dominant cause of death in the world (Alwan, 2011), studying predictors of this serious ailment is imperative. The development of cardiovascular disease can be powerfully predicted by frequent or chronic reductions in the variation of time between successive heart beats (i.e., by reductions in so-called heart rate variability or HRV; Bosma et al., 1998; OrthGomér et al., 2000; Matthews and Gump, 2002; Rosengren et al., 2004; Kivimäki et al., 2006) as well 
as to precede the development of several risk factors, like hypertension, high cholesterol, diabetes, and immunological markers of pathogenic states (Thayer and Lane, 2007; Thayer et al., 2010; Jarczok et al., 2019). The risk of negative cardiovascular events may be increased by as much as 32$45 \%$ by low HRV (Hillebrand et al., 2013) and a potential cause of such HRV reductions may be prolonged exposure to psychological stress (McEwen, 2001). Stress can be considered to be a complicated, multidimensional phenomenon that may be strongly related to the consistency of a person's emotional response to life events (e.g., with happiness, anger, etc., Lazarus, 1993).

The relationship between stress and changes in physiological parameters that are not due to changes in physical activity was demonstrated first by Blix et al. (1974) in helicopter pilots during take-off. Our lab has recently developed a technique to detect episodes of reduced HRV in ambulatory participants (Verkuil et al., 2016), and to relate these physiological episodes to the participants' self-reported episodes of psychological stress and worrying (Verkuil et al., 2016; Brown et al., 2018). We had participants wear an ECG sensor for $24 \mathrm{~h}$, as they went about their daily doings. Each experimental session started with a short calibration period in the lab, during which participants engaged in four classes of energy-expending physical activity that might also be performed during a regular day: standing, cycling, climbing stairs, and lying down. The participants' HRV was measured during each of these classes of activity. We used these data to compute an HRV baseline during various levels of physical activity ${ }^{1}$. Some studies have excluded all periods of high physical activity (Brosschot et al., 2007; Pieper et al., 2007, 2010), and/or have identified epochs of non-movement by using accelerometer readings (Sowder et al., 2010), but the interrelatedness of activity levels and HRV (Rennie et al., 2003) makes it imperative to take levels of physical activity into account when studying HRV. Research has consistently shown that during episodes of physical activity, heart rate increases are associated with HRV decreases (for an extensive review, see Michael et al., 2017). A number of other factors influence HRV (reviewed in Fattison et al., 2016), and we therefore only include participants who neither smoked, nor were on antihypertensive or cardiological medication like beta-blockers (also see the Discussion). Our approach enabled us to estimate the amount of HRV that is not due purely to physical activity (i.e., additional physiology, in the current study, HRV: the term additional was coined by Blix et al., 1974) and this, in turn, allowed us to examine the relationship between psychological factors such as stress and emotions and physiological activity.

In the Verkuil et al. (2016) study, the HRV and physical activity data that were collected during a calibration period in the lab, were used to compute personalized algorithms for each participant, which could then be utilized to detect episodes associated with reductions in additional HRV. Given that these episodes of reduced additional HRV were not associated with concurrent levels of movement, they can therefore considered

${ }^{1}$ By physical activity, we mean bodily movement of various intensities (e.g., casual walking vs. climbing stairs). to be related to psychological stress (Myrtek et al., 2005). Furthermore, participants were prompted hourly to fill out some questionnaires on mobile phones while wearing their ECG sensors. The questionnaires assessed whether participants had experienced stress or worry during the previous hour, enabling us to associate physiological stress markers and psychological stress markers.

Techniques to estimate additional physiology are involved methods that have only occasionally been used in ambulatory emotion-related studies; furthermore, heart rate was typically studied instead of HRV and, crucially, no individualized algorithms were utilized (Myrtek and Brügner, 1996; Myrtek, 2004; Myrtek et al., 2005; Ebner-Priemer et al., 2007; Prill and Fahrenberg, 2007). Verkuil et al. (2016) developed a two-step process to detect episodes of additional HRV reductions: first, the relationship between HRV and movement (i.e., walking, cycling, etc., all expressed as acceleration) was formalized by fitting an inverse regression model to the data that were acquired during the calibration period. This was done separately for each participant. The obtained model parameters were then utilized to detect episodes of reduced additional HRV (see below, as well as Verkuil et al., 2016, for more detail). In a follow-up study, we then related these episodes of reduced additional HRV to episodes of worry or stress that were self-reported by participants (Brown et al., 2018). In the same study, we explored alternative methods to that used by Verkuil et al. (2016). All of these methods, which are detailed in the "Materials and Methods" section below, are based on a similar principle: the construction of an inverse regression model that quantifies the relationship between HRV and movement. However, these alternative methods use different subsets of the data to compute those inverse regression models. For example, instead of using data obtained during a calibration phase, as Verkuil et al. (2016) did, in one of our alternative methods, we used the first $10 \mathrm{~min}$ worth of data from every available hour to create our inverse regression model. One alternative method seemed particularly promising: simply using all available data (in the Verkuil et al., 2016, study, that yielded $24 \mathrm{~h}$ worth of data) for a given participant led to a considerably better match between physiological episodes of reduced additional HRV and self-reported psychological episodes of worry and stress than using the data from the lab calibration.

We have evaluated these alternative methods by reanalyzing data and found promising results, so further tests on a dataset that was acquired for that specific purpose was in order. We have therefore acquired data for six participants, who were subjected to three $24 \mathrm{~h}$ test sessions. Not only did this allow us to test our methods on a dedicated dataset, but it also allowed us to evaluate two more methods to further explore the optimal method to estimate reductions in additional HRV. First of all, we have added a class of activity to Verkuil et al. (2016) calibration phase in the lab: participants were required to read a complicated text out loud and they had to clench their fists and tense their shoulders for 3 min. We expected that reading out loud while the experimenter listened would evoke feelings of stress in the participants, while clenching their fists and tensing their shoulders might reduce their HRV, which is common for such isometric activity (i.e., clenching muscles without actually moving; Stewart et al., 2007). 
Incorporating this "stress-induction" class of activity into the lab calibration phase may therefore improve the sensitivity to detect episodes of reduced additional HRV in the method that used lab calibration data. Having access to $72 \mathrm{~h}$ worth of data for every participant allowed us to introduce and explore one further method: it might be possible to use the data of day $n$ to detect episodes of reduced additional HRV in day $n+1$. This method has the advantage of avoiding "double dipping" into the data by using an inverse regression model that was established on one dataset (i.e., one $24 \mathrm{~h}$ period) to detect episodes in another dataset (i.e., another $24 \mathrm{~h}$ period) of the same participant.

Given the modest sample size of the current study-although the results were clear and in line with earlier work-we have also reanalyzed a dataset from our lab, which also included $72 \mathrm{~h}$ worth of data for participants (Versluis et al., 2018). However, we were unable to test all of our hypotheses in this dataset alone, as the participants in this dataset were not subjected to the laboratory calibration phase that was used by Verkuil et al. (2016).

The goal of the current paper is to further explore the optimal way to study and quantify the relationship between additional HRV reductions and movement, to learn more about this powerful and important predictor of cardiovascular disease. We will compare the performance of the various methods in a number of ways and, crucially, we will determine which of the methods is best able to detect episodes of reduced additional HRV, as demonstrated by a high correspondence between such method-identified physiological episodes and participantreported episodes of psychological stress and worrying. Given our previous findings, we expect the method that used all available data for a given session to perform best. The method that used the first $10 \mathrm{~min}$ of three consecutive hours may also perform well, based on previous findings. Our two new methods (extended lab calibration that incorporated stress induction and using the data of day $n$ to detect episodes of reduced additional HRV in day $n+1)$ may, in turn, outperform these two methods.

\section{MATERIALS AND METHODS}

\section{Study Design}

We reanalyzed one dataset (Versluis et al., 2018, institutional review board approval number 4689348773) and we acquired data from six new participants to evaluate a number of methods to optimally detect episodes of reductions in additional HRV.

\section{Setting}

Participants for the new dataset were tested at Leiden University in 2015 and 2016. Participants were recruited via posters in the building and through Leiden University's digital participant recruitment system. For the Versluis et al. (2018) dataset, please see the section "Materials and Methods" section in the relevant paper.

The six new participants we tested for this study were invited into the lab, were fitted with an ECG sensor, mounted on a chest strap, and were provided with an Android-based Motorola Razr cell phone which prompted them once an hour, at random moments within that hour, to fill out a set of psychological questionnaires about whether they had experienced stress and worry during the past hour, and how long these episodes had lasted (for more information, see Verkuil et al., 2016, whose procedure was followed). Participants then undertook the following types of (physical) activity, without breaks in between: (1) sitting down for 3 min while watching a relaxing video; (2) standing up for 3 min while counting in steps of two (to keep participants' minds off of ruminating); (3) lying down for 3 min while counting in steps of two; (4) cycling on a stationary bike for $3 \mathrm{~min}$; (5) sitting down, clenching one's fists and tensing the shoulders for $3 \mathrm{~min}$; (6) reading a text on the history of Leiden University out loud for 3 min, while the experimenter listened; (7) climbing four flights of stairs (63 steps). Two types of activity were added to the Verkuil et al. (2016) lab calibration: clenching one's fists and tensing the shoulders, which was expected to reduce HRV, and reading a complicated text out loud, which was expected to evoke feelings of stress. After engaging in all of these types of activity, participants were sent home, with instructions to wear the ECG monitor for $24 \mathrm{~h}$ and fill in the hourly questionnaires on the smartphones.

\section{Participants}

We tested six participants (four females, mean age 26.5 years), who were each subjected to three 24 -h test sessions. Unfortunately, one 24-h dataset was not recorded by the ECG sensor due to technical reasons; this left us with five complete 72 - $\mathrm{h}$ datasets and one dataset of $48 \mathrm{~h}$. We have included the participant for whom we had $48 \mathrm{~h}$ worth of data in our analyses and considered one $24 \mathrm{~h}$ dataset to be statistically "missing data" that were not replaced. We have also reanalyzed data from Versluis et al. (2018). Due to technical reasons which have resulted in noisy data, we were only able to analyze 5 participants from the control condition of the study from Versluis et al. (2018) (all females, mean age 26 years), which obviously limits the power of the concomitant analyses; we consider this to be a set of exploratory analyses. All participants signed informed consent before being included and they were financially compensated according to Leiden University's policy for the remuneration of participants. For both studies, participants who smoked, or who were on antihypertensive or cardiological medication (like beta-blockers) were not allowed to participate in the studies.

\section{Variables}

In the current study, we have evaluated six alternative methods to the additional HRV detection method that was described by Verkuil et al. (2016). All of these methods used a similar approach: an inverse regression model was fitted for every individual to quantify the relationship between HRV, expressed as the root mean squares of successive differences, RMSSD ${ }^{2}$,

\footnotetext{
${ }^{2}$ There are multiple methods to estimate HRV either in the time or frequency domains. We selected this specific method because it is commonly used (Task Force of The European Society of Cardiology and The North American Society of Pacing, and Electrophysiology, 1996; Berntsen et al., 2017, p. 196) and recently, single RMSSD values were shown to be powerful predictors of health risk factors in a very large sample (Jarczok et al., 2019).
} 
and movement, which was expressed as acceleration in $g$ (the averaged acceleration in three axes), according to Eq. 1.

$$
\text { Expected } \mathrm{RMSSD}_{i, j}=B 0_{i}+\frac{B 1_{i}}{\text { acceleration }_{i, j}}
$$

In this inverse regression model, an expected RMSSD value for participant $i$ at 30 -s sampling interval $j$ was computed as the sum of the value of RMSSD while no acceleration was present (i.e., the intercept, $B O_{i}$ ) and the change in RMSSD that was due to acceleration (i.e., the slope, $B 1_{i}$ ). The standard error of the mean of RMSSD was also computed, to be used in later computations (see section "Data Sources/Measurement" below).

RMSSD was computed for 30-s intervals throughout the entire 24-h test session and was then averaged over samples that spanned different amounts of time, depending on the particular method used (see below). $g$, as used in this study, has been demonstrated to be a valid method to measure movement, especially walking, jogging, sitting, and lying down (Lugade et al., 2014). We then fitted the inverse regression model to a subset of all the data points that were available for a given participant; for example, Verkuil et al. (2016) fitted such a model to the HRV and movement data that were acquired during a laboratory calibration period while participants performed various activities. The parameters from these regression models were then utilized, for each individual participant, to predict HRV levels as a function of movement levels. Whenever actual HRV levels fell two standard errors below predicted HRV levels, and such a difference lasted at least 7.5 consecutive minutes ${ }^{3}$, we considered this to represent an episode of decreased additional HRV (formulae are presented in Verkuil et al., 2016). In line with Verkuil et al. (2016), if multiple such episodes were identified within a given hour, we only used the first episode that was detected within that hour in further analyses.

\section{Data Sources/Measurement}

All 24-h ECG data and movement data were collected with an ecgMove sensor (ECGMove 3, Movisens, GmbH, Karlsruhe, Germany). Data were processed offline in Movisens DataAnalyzer version 1.12 (preprocessing and artifact rejection were described in Verkuil et al., 2016); the Data-Analyzer software uses automated algorithms to detect and remove artifacts in the data. Data were then analyzed in MATLAB ${ }^{\mathrm{TM}}$ (MathWorks, Natick, Massachusetts). Analysis scripts are available from the corresponding author. The methods that were used to detect episodes of reduced additional HRV are described next.

\section{Method 1: First 10 Min of Every Hour}

This method, described previously in Brown et al. (2018), computed inverse regression models based on the first $10 \mathrm{~min}$

\footnotetext{
${ }^{3}$ The Verkuil et al. method was developed in a first attempt to identify episodes of reduced additional HRV in an ambulatory setting, and it has guided the current work. Requiring actual HRV to be two standard errors below the predicted HRV for $7.5 \mathrm{~min}$ is a carefully considered but potentially seemingly arbitrary decision. Verkuil et al. reasoned that two standard errors would provide a pronounced enough difference between the two variables, and that $7.5 \mathrm{~min}$ would be enough time to qualify a period as representing worrying or stress. For a discussion of these decisions, see Brown et al. (2018).
}

of every hour for which data were collected. For example, this yielded 24 separate inverse regression models if $24 \mathrm{~h}$ worth of data were available. Episodes of reduced additional HRV (see above) were detected for every hour, using the model parameters for that specific hour. An advantage of this method is that episodes of reduced additional HRV within a given hour were detected with a model that was based on data from that specific hour. This "double dipping" is also this method's disadvantage: (part of) the data from hour $n$ were used to detect additional HRV episodes in hour $n$. Such episodes were also looked for in the first $10 \mathrm{~min}$ of data, which was the time period used to construct the inverse regression model that was utilized for this detection process in the first place. Nevertheless, this appears to be a fairly minor concern: should an episode of reduced additional HRV be identified in the first 10 min of a given hour, then there seems to be no empirical reason to question whether a person actually experienced worry or stress during those $10 \mathrm{~min}$ (also see section "Discussion”).

\section{Method 2: Full Dataset}

This method used the entire period for which data were collected during a given test session to compute an inverse regression model. For example, if $24 \mathrm{~h}$ worth of data were available, a single inverse regression model was computed, based on that entire $24-\mathrm{h}$ period. The parameters from this model were then used to identify episodes of reduced additional HRV separately for every hour in that dataset. The inverse regression models in this method are based on a large number of data points, rendering these models more robust than models based on just 10 min worth of data, which is an advantage this method offers. This advantage is slightly offset by the levels of movement and HRV being averaged over the total available time period and them not being modeled separately for each hour. This method has been described previously in Brown et al. (2018).

\section{Method 3: First 10 Min of Three Consecutive Hours}

As discussed previously in Brown et al. (2018), this method computed an inverse regression model based on the first $10 \mathrm{~min}$ worth of data for three consecutive hours, which allowed us to compensate for fluctuations over time in movement or HRV. In this method, every regression model was therefore based on 30 min worth of data. The inverse regression models for the first and last hours of a dataset were based on the first $10 \mathrm{~min}$ of the first and second hours of the dataset and on the first $10 \mathrm{~min}$ of the penultimate and final hours, respectively. So, if $24 \mathrm{~h}$ worth of data were available, we computed 24 separate inverse regression models. An advantage of this method is that changes in HRV or movement levels over three consecutive hours were taken into account. Furthermore, the inverse regression models were based on an average of the first $10 \mathrm{~min}$ of three consecutive hours, which leads to more reliable model parameter estimations than 10 using only $10 \mathrm{~min}$ worth of data would (cf. Method 1, above). However, using HRV and movement data from three consecutive hours is also a disadvantage: more data may be used to compute inverse regression models, but movement and HRV levels are autocorrelated over time, which would reduce variation in these levels over three consecutive hours. 


\section{Method 4: Movement Level Bins}

This method, discussed previously in Brown et al. (2018), used the natural variation in a participant's movement levels throughout the day. We therefore binned movement data based on quartiles that were defined per participant, thus creating four bins that classified levels of movement that ranged from relatively very low to relatively very high. Each bin contained 5 consecutive minutes' worth of data: a participant's level of movement within these $5 \mathrm{~min}$ had to be in between two quartiles to be assigned to a specific bin. Given our desire to identify all four bins for as large a number of participants as possible, we chose to base bins on periods of five consecutive minutes; making bins wider (i.e., encompassing more time), would attenuate the number of participants for whom all four bins could be identified (see below). Our analysis was restricted to the $5 \mathrm{~min}$ bin of every movement class that occurred first. For example, if four clusters of quartile-1 movement were identified for a given participant, only the first cluster was used in subsequent analyses, so that each bin contained the same amount of data for each participant (e.g., not $5 \mathrm{~min}$ for one participant and $80 \mathrm{~min}$ for another participant). Inverse regression models were based on all available quartile data, so a maximum of 20 min worth of data. By representing four levels of movement in the inverse regression models, this method takes variations in an individual's movement into account, which is an advantage that is not offered by methods that only use the data from the first $10 \mathrm{~min}$ of every hour, which are, of course, unlikely to contain each possible level of movement by mere chance. Another advantage of this method is that it only requires the computation of a single inverse regression model, as opposed to methods that require models for every hour of data. Unfortunately, not all four bins could be identified in one of the six newly tested participants. Of course, this participant did have movement data that fell between two quartiles, but s/he simply did not have 5 consecutive minutes' worth of such data. However, the other three bins could be identified in this participant, yielding 15 min worth of data that were usable to compute an inverse regression model. The same applied to the five reanalyzed participants from the Versluis et al. dataset. In a way, this activity bin method is comparable to the laboratory-based method of Verkuil et al. (2016), which was based on four predetermined physical activity categories: lying down, standing, cycling and climbing stairs. However, the activity bin method it is data-driven and does not depend on a laboratory-based calibration data, which might not be available to interested researchers, and this renders the activity bin method more versatile.

\section{Method 5: Extended Calibration}

This method is highly comparable to the original method by Verkuil et al. (2016): we computed an inverse regression model on the data acquired during the lab calibration, but we added two types of activity: reading a complicated text out loud and clenching one's fists while tensing one's shoulders. We expected these two activities to be stressful and to lower HRV, respectively. Therefore, adding these two types of activity may lead to the formulation of a model that has a better fit than Verkuil et al. (2016) original models, as (simulated) stress and an HRVlowering procedure are now also included in the calibration phase. A disadvantage of this method is that it is difficult to find a text that is difficult to read for all participants; although most of our participants stumbled over such words as "string galvanometer" and the Latin names of ancient professors (e.g., Jacobus Arminius, Daniel Heinsius, etc.), other participants read through these texts with few issues.

\section{Method 6: Next-Day Prediction}

An important question following the work that was reported by Brown et al. (2018) was whether it might be possible to use the inverse regression model of day $n$ to identify episodes of reduced additional HRV in day $n+1$. This method was performed on a subset of all available data: we used all the data for a participant's first testing day to detect episodes of reduced additional HRV in his or her second day, and all the data from his or her second day to detect episodes in the third day. The advantage of this method is that there is no double dipping into the data, as the model used to detect episodes of reduced additional HRV in a given day is based on data from a completely different day. The obvious disadvantage was that no episodes of reduced additional HRV could be detected in the first test session $(n)$, because that would require an inverse regression model to be computed for day $n-1$; theoretically, one could use the data from, for example, the last testing day for that purpose. Given our specific interest in the efficacy of "next-day" predictions, we have not explored that option further (cf. section "Discussion").

\section{Episodes Detected During Sleep}

Occasionally, our methods identified episodes of decreased additional HRV during a participant's sleep. If actual measured HRV is well below expected HRV levels, this can happen, but clearly, such a phenomenon cannot be due to conscious psychological stress during a participant's sleep. Having said that, for methods in which the inverse regression model was calculated based on data acquired during waking periods, these episodes of decreased additional HRV may have meaning, given that an association between low sleeping HRV and preceding stress has been reported (e.g., Hall et al., 2004; Brosschot et al., 2007). This relationship has been suggested to reflect unconscious stressrelated cognition (Brosschot, 2010; Brosschot et al., 2010). Given that nocturnal HRV was not the primary focus of this paper, we have chosen not to include such episodes in our analyses.

\section{Bias}

Given that there was no experimental manipulation in this study, experimenter bias toward participants was minimized. Given that all analyses were automatized through scripts, and the outcome variables were the number of episodes of reduced additional HRV that were detected by those scripts, experimenter bias and subjectivity were minimized.

\section{Study Size}

Following the work reported by Brown et al. (2018), we continue to explore and refine our methods to detect episodes of reduced additional HRV. Our choice of sample size reflects this exploratory nature (also see the Discussion). 


\section{Quantitative Variables}

The handling of quantitative variables was described in detail under Data sources/management.

\section{Statistical Methods}

The current paper's objective was to evaluate the efficacy of the six methods described above in detecting episodes of reduced additional HRV. To this end, we have compared these methods in three ways, following the strategy introduced in Brown et al. (2018). Two of these comparisons served to demonstrate the variation in number of episodes of reduced additional HRV that was identified by each method. Firstly, to explore the variation in numbers of identified episodes of reduced additional HRV, we compared the number of episodes each method detected by using repeated-measures analyses of variance (ANOVAs) with test day and the different additional HRV estimation method (e.g., first $10 \mathrm{~min}$ of every hour, etc.) as within-subjects factors. We then computed Pearson correlations between the numbers of episodes that were detected in three $24 \mathrm{~h}$ periods, to test the temporal reliability of these methods. These two analyses have been performed for both datasets analyzed here.

In our newly acquired data, participants reported once an hour whether they had been stressed or worried during the past hour. We then performed a vital analysis, in which the onsets of participants' self-reported episodes of psychological stress and worry were compared to the onsets of the episodes of reduced additional HRV that were identified by each of the methods. The first two analyses charted the distribution of the number of physiological episodes of reduced additional HRV that the different methods identified, as well as the temporal reliability of each method, but this key comparison revealed true episodes of reduced additional HRV by demonstrating to what extent these physiological events matched up with psychological events of stress and worry. We have therefore computed, separately for every method and for every available hour worth of data, the percentage of participants that had matches between episodes of reduced additional HRV and selfreported stress and worry episodes. We then calculated the average percentage of such matches within a given method. These three comparisons are expected to assess the quality of each of the methods discussed here and to ascertain which of these methods seems to be the best alternative to the laboratory calibration method presented by Verkuil et al. (2016).

\section{RESULTS}

\section{Reanalysis of Versluis and Colleagues Data}

As there was no calibration phase in the study by Versluis et al. (2018), we utilized four methods to identify episodes of additional HRV: we used the first $10 \mathrm{~min}$ of every hour, the full dataset of $24 \mathrm{~h}$, the method that used a combination of the first $10 \mathrm{~min}$ of three consecutive hours, and the method that used activity type bins. Some methods identified more reduced additional HRV-episodes than others, as presented in Table 1.
TABLE 1 | Mean number of additional HRV episodes $(S D)$ identified in three test days of $24 \mathrm{~h}$ each.

\begin{tabular}{lcccc}
\hline & $\begin{array}{c}\text { First } \mathbf{1 0} \text { min of } \\
\text { every hour }\end{array}$ & $\begin{array}{c}\text { Full } \mathbf{2 4} \mathbf{h} \text { of } \\
\text { first test day }\end{array}$ & $\begin{array}{c}\text { First } \mathbf{1 0} \text { min } \\
\text { of } \mathbf{3} \mathbf{~ h}\end{array}$ & $\begin{array}{c}\text { Activity } \\
\text { type bins }\end{array}$ \\
\hline $\mathrm{T} 1$ & $9.6(2.6)$ & $16.4(2.1)$ & $12.4(1.1)$ & $5.2(3.3)$ \\
$\mathrm{T} 2$ & $7.8(3.7)$ & $14.6(3.1)$ & $12.8(2.9)$ & $11.0(6.1)$ \\
$\mathrm{T} 3$ & $6.8(1.9)$ & $13.6(2.0)$ & $10.4(1.1)$ & $14.0(2.5)$ \\
$\bar{X}$ & 8.1 & 14.9 & 11.9 & 10.1 \\
\hline
\end{tabular}

Calibration time period was also included in detection periods. Every method detected additional HRV episodes in all five analyzed participants.

A repeated-measures ANOVA with test day and estimation method as within-subjects factors revealed a significant difference between methods, $F(3,12)=25.5, p<0.0005, \eta_{p}^{2}=0.72$. This effect suggested that the method that used the full dataset identified the largest mean number of episodes of reduced additional HRV (14.9), while the methods that used the first $10 \mathrm{~min}$ of every hour identified the lowest number of episodes (8.1). There was no reliable effect of test day, $F(2,8)=0.14$, $p=0.87, \eta_{p}^{2}=0.03$, but test day and method interacted, $F(6,24)=10.0, p<0.0005, \eta_{p}{ }^{2}=0.72$. Three of the four methods investigated here seem to identify relatively robust numbers of additional HRV episodes over time, but this interaction seems to be driven by the outlying observation in the first test day for the method that used activity class to detect reduced additional HRV episodes (5.2 episodes in the first session vs. 11.0 and 14.0 episodes in the second and third sessions, respectively). This may be an artifact of the low power of the analyzed sample, as there is no theoretical reason to assume this method would identify a lower number of additional HRV episodes in one of the three $24 \mathrm{~h}$ periods that were analyzed. Furthermore, pairwise comparisons suggested that the only significant differences in identified additional HRV episodes were those between the first and second test day for the activity class method, $t_{4}=3.7, p=0.02$ and between the first and third times series for the activity class method, $t_{4}=4.5, p=0.01$. All other differences were not significant (all ps $>0.06$ ).

To further explore the reliability of the methods over time, we computed Pearson correlations between the average number of episodes detected over time, separately for every method. The largest correlation observed was the one between the number of episodes detected in the second and third test day for the method that used a combination of $10 \mathrm{~min}$ from three consecutive hours, $r=-0.97, p=0.008, \mathrm{CI}_{95}=[-0.99,-0.61]$. The correlation between the first and second test day for the method that used activity type was also large, $r=0.88, p=0.051, \mathrm{CI}_{95}=[-0.01,0.99]$. The lack of statistically robust correlations is likely due to the low power of the analyzed sample: we therefore urge the reader to interpret these correlations as effect sizes without reliance on the associated $p$-values; excepting the activity class method, all methods seem to identify numerically relatively similar numbers of additional HRV episodes over time. Note that the method that used the full dataset to identify episodes of reduced HRV, which was associated with a good match between episodes of self-reported stress and worry and method-identified episodes of additional HRV in other work (Brown et al., 2018), also 
appears to provide a stable estimate of additional HRV reduction episodes over time (all $r$ s between 0.70 and 0.80 ). Unfortunately, the crucial test, in which self-reported psychological stress and worry episodes are related to method-identified physiological episodes of reduced additional HRV, could not be performed for the Versluis et al. (2018) dataset, as there was no hourly selfreported stress or worry data available for these participants. This was a powerful motivation to test additional participants.

\section{New Data to Test All Methods}

We tested six new participants to further evaluate all six methods and to corroborate findings reported elsewhere (Brown et al., 2018). We first compared the numbers of episodes of reduced additional HRV that were identified by every method. Given that we could only analyze two of the three days' worth of data for the method that used the data of day $n$ to detect episodes of reduced additional HRV in day $n+1$ (because there is no data before day 1, only days 2 and 3 could be analyzed with this method), we have evaluated that method separately. We therefore first performed a repeated-measures ANOVA with test day and estimation methods as within-subjects factors, which suggested that the various methods identified different numbers of episodes of reduced additional $\operatorname{HRV}, F(5,20)=6.6, p=0.001$, $\eta_{p}^{2}=0.62$

As can be seen in Table 2, Verkuil et al. (2016) lab calibration method detected the lowest number of episodes (3.6), while the method that used the full dataset identified the largest number of episodes (11.4). Interestingly, pairwise comparisons revealed no significant differences whatsoever, suggesting that the different methods each identified reliable numbers of episodes of reduced additional HRV over time. Furthermore, it is interesting to note that, once again, the method that used the full dataset identified the largest number of episodes overall. There was no reliable effect of test day on number of episodes detected, nor did method and test day reliably interact ( $p$ s of 0.29 and 0.49 , respectively).

We then computed Pearson correlations between numbers of episodes detected over time, again, separately for every method: we have, once more, treated these correlations as effect sizes. The largest correlation was for the numbers of episodes detected in test days 1 (6.1 episodes) and 3 (6.8 episodes) for the method that used bins of activity type, $r=0.95, p=0.01, \mathrm{CI}_{95}=[0.61,0.99]$. The majority of correlations (11 out of 15) exceeded $r=0.40$. Clearly, these correlations are based on a low-powered dataset. Furthermore, these low correlations are not necessarily indicative of poor temporal reliability of the methods evaluated here, as participants could simply have experienced different numbers of episodes of reduced additional HRV on the various days they were tested on.

We performed another repeated measures ANOVA like the one described above, but we now incorporated the final method, which used the data from day $n$ to detect episodes of reduced additional HRV in day $n+1$. Because this method only allowed us to analyze data from the second and third days of testing, we have only incorporated these two test days for every method analyzed. This analysis revealed a reliable difference in number of identified episodes of reduced additional HRV; even taking just the second and third test day for every participant into account, the lab calibration method by Verkuil et al. (2016) still identified the lowest number of episodes (3.5), while the method that used the data of day $n$ to detect episodes in day $n-1$ identified the largest number of episodes (11.2; 9.4 episodes during the second day and 13.0 during the third), $F(5,20)=6.7, p<0.0005$, $\eta_{p}{ }^{2}=0.63$. It is interesting that the latter method identified marginally more episodes than the method that used the full dataset (10.9). There was no effect of test day, nor did method and test day interact ( $p$ s 0.59 and 0.41 , respectively).

\section{Associations With Worries and Stress}

The crucial test for any of these methods is to see how well the physiological episodes of reduced additional HRV they detected correspond with participants' self-reported episodes of stress and worrying. After all, the detected physiological events are only of real interest if they actually coincide with-so, representknown episodes of worrying or stress: only then do they actually signify reductions in additional $\mathrm{HRV}$, as opposed to possibly random physiological events in the data that may not have a clear psychological cause. Participants reported and average of 2.8 $(S D=3.2)$ episodes of worrying and/or stress.

To this end, we first calculated the percentage of participants with at least one match between an episode of method-identified reduced HRV and an episode of self-reported worrying or stress in their data. These percentages were calculated separately for every method. As presented in the first row of Table 3, certain methods were characterized by considerably higher match percentages than others.

For example, the method that utilized the full dataset was associated with an $85.7 \%$ match between participants' selfreported stress and worrying episodes and the episodes of reduced additional HRV identified by this method; this method was also found to be very promising in other work (Brown et al., 2018). Interestingly, the method that used the first $10 \mathrm{~min}$ of three consecutive hours to detect episodes of reduced additional

TABLE 2 | Mean number of additional HRV episodes (SD) identified in three test day of $24 \mathrm{~h}$ each.

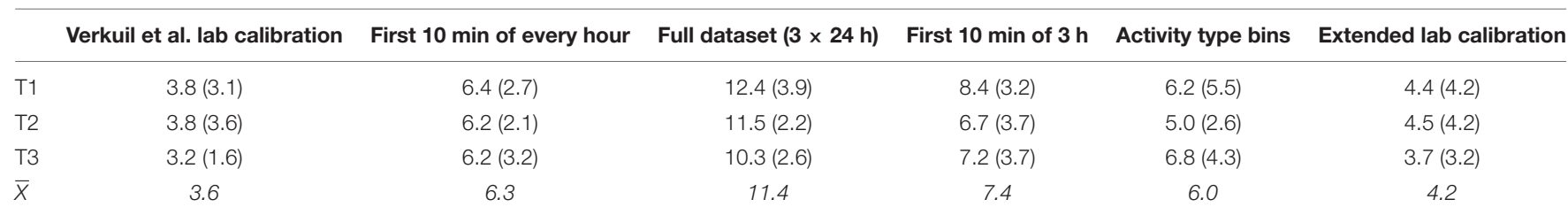

Calibration time period is also included in detection periods. Every method detected additional HRV episodes in all five analyzed participants. The bottom row contains the number of identified episodes averaged over test day. 
TABLE 3 | Matches between method-identified episodes of reduced additional HRV and self-reported episodes of stress and worry (expressed as percentages).

\begin{tabular}{|c|c|c|c|c|c|c|c|}
\hline & $\begin{array}{c}\text { Verkuil et al. lab } \\
\text { calibration (\%) }\end{array}$ & $\begin{array}{l}\text { First } 10 \text { min of } \\
\text { every hour }(\%)\end{array}$ & $\begin{array}{l}\text { Full dataset } \\
(3 \times 24 \text { h) }(\%)\end{array}$ & $\begin{array}{l}\text { First } 10 \text { min of } \\
3 \mathrm{~h}(\%)\end{array}$ & $\begin{array}{l}\text { Activity type } \\
\text { bins (\%) }\end{array}$ & $\begin{array}{c}\text { Extended lab } \\
\text { calibration (\%) }\end{array}$ & $\begin{array}{c}\text { Next day } \\
\text { detection (\%) }\end{array}$ \\
\hline Participants with $\geq 1$ match & 42.9 & 78.6 & 85.7 & 85.7 & 78.6 & 42.9 & 60.0 \\
\hline Mean percentage of matches & 50.9 & 65.5 & 70.1 & 73.6 & 59.8 & 53.0 & 68.7 \\
\hline
\end{tabular}

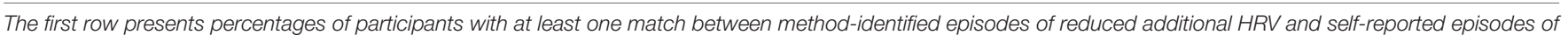

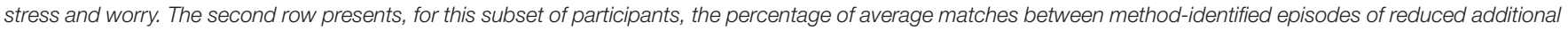
HRV and self-reported episodes of stress and worry.

HRV was associated with an identical percentage of matches. The Verkuil et al. (2016) lab calibration method was associated with a match of $42.9 \%$. This would suggest that the methods that used all available data for a given test session and that used a combination of $10 \mathrm{~min}$ of three consecutive hours' worth of data are more sensitive in detecting actual episodes of reduced additional HRV than Verkuil et al. (2016) lab calibration method, which we considered to be a golden standard. Indeed, earlier work (Brown et al., 2018) had identified the method that used a combination of $10 \mathrm{~min}$ of three consecutive hours to be a good "runner-up" to the method that used the full dataset.

The percentages reported above merely indicate how many participants had at least one match between method-detected episodes of reduced additional HRV and self-reported stress or worry episodes. A crucial next step is to compute the percentage of matches between method-detected episodes of reduced additional HRV and self-reported stress or worry episodes within the participants with at least one such match. Those percentages reveal how well, on average, each method was able to identify physiological episodes of reduced additional HRV that match and therefore represent self-reported episodes of stress or worry (provided that a participant had at least one such match). These percentages are listed in the second row of Table 3. Once more, the methods that used the full dataset and a combination of $10 \mathrm{~min}$ of three consecutive hours appear the most promising, with an average match between methodidentified episodes of reduced additional HRV and self-reported stress worry of 70.1 and $73.6 \%$, respectively. Although the method that used the data of day $n$ to identify episodes of additional HRV reduction in day $n+1$ performed relatively well, with a matching percentage of $68.7 \%$, this method was only able to detect matches in $60.0 \%$ of all tested participants. The extended calibration method performed similarly to the Verkuil et al. (2016) lab calibration method, which is not surprising, given that it is based on that method. Taken together, all of the results above suggest that using the full dataset to create an inverse regression model, which will then be used to detect episodes of reduced additional HRV, or using a combination of the first $10 \mathrm{~min}$ of three consecutive hours' worth of data to create such an inverse regression model appear to be the most promising methods. These two methods were associated with the best overall match between additional HRV physiology and self-reported stress and worry in the highest number of participants. Interestingly, all of the methods evaluated here identified more physiological reduced additional HRV episodes than that participants reported episodes of stress or worrying. The methods identified more episodes, on average, than participants reported in $85.2 \%$ of cases.

\section{DISCUSSION}

The goal of the current paper was to evaluate several methods that can be used to identify episodes of reduced additional HRV and to identify the optimal method. As in previous work (Brown et al., 2018), we found that using every data point available in a given dataset appears to be a very promising method. Interestingly, the method that was identified to be a "runner-up" in our earlier study, slightly outperformed the method that used the full dataset in the current experiment: using the first $10 \mathrm{~min}$ of three consecutive hours to detect episodes of reduced additional HRV also led to a very good match between such physiological episodes and self-reported episodes of psychological stress and worry. Finally, the method that used the first 10 min of every available hour to detect episodes of reduced additional HRV also seemed to perform well enough to be considered an interesting option. The advantage of all three of these methods is that they do not rely on a calibration phase in the laboratory, which facilitates the identification of episodes of reduced additional HRV for researchers who do not have access to the calibration procedure introduced by Verkuil et al. (2016).

Two new methods were evaluated here: introducing an additional class of activity during the calibration phase, and using the data from day $n$ to detect episodes of reduced additional HRV in day $n+1$. These two methods did not perform as well as the three methods described above: the extended calibration method performed similarly to the Verkuil et al. (2016) calibration method; the method that used data from day $n$ to detect episodes in day $n+1$ seemed the more promising of the two new methods, and it may therefore warrant further exploration. One problem with this method is that it requires the acquisition of at least two sessions' worth of data, while episodes of reduced additional HRV are detected in only one of those sessions. Of course, one could devise ways to utilize the data of the "lost" session, for example by using the data of the last available test session to create an inverse regression model and to then use the resulting model parameters to detect episodes of reduced additional HRV in the first available test session. Clearly, this would complicate the method, as one would no longer be using a predictive model to detect episodes, but one would be using a kind of retroactive "prediction." For this reason, and given the method's overall unpromising level of performance, we have not explored this method beyond simply analyzing the data from the second and third test sessions in this paper. 
One issue that we have commented on before (Brown et al., 2018), but that also characterizes the data of the six participants we have tested here, is the discrepancy in numbers of self-reported episodes of psychological worry and stress and method-identified physiological episodes of reduced additional HRV. The numerous methods invariably identify (occasionally, considerably) larger numbers of episodes of reduced additional HRV than that participants report episodes of stress and worry. For example, on average, the method that used the full dataset identified 11.4 episodes of reduced additional HRV, averaged over three test sessions, while participants reported 2.8 episodes of psychological stress or worry, averaged over three test sessions. It seems implausible that this effect is caused by technological glitches in the cell phones that were used to record participants' responses to questionnaires about their current psychological state. This leaves two explanations: either participants underreported episodes of stress and worry for any number of reasons (social expectations, shame, forgetfulness, etc.), or the episodes of reduced additional HRV that were detected by the various methods but that were not matched by self-reported stress and worrying episodes were due, at least in part, to unconscious stress. The latter option is corroborated by other work from our lab (Brosschot, 2010; Brosschot et al., 2010).

Another explanation could be that positive psychosocial events evoked these yet unexplained reductions in HRV. Indeed, previous work has shown that such events can also lead to increases in heart rate and therefore to concomitant reductions in HRV (see, e.g., Jacob et al., 1999). However, sustained increases in heart rate have been shown to be associated with events of negative valence only (Brosschot and Thayer, 2003). Given that the duration of our additional HRV reduction detection episodes was $7.5 \mathrm{~min}$, and given that Brosschot and Thayer showed that heart rate already starts to decrease (and therefore, HRV starts to increase) after $5 \mathrm{~min}$ of being presented with a positively-valenced stimulus, we do not believe that additional HRV reductions due to positive psychosocial events provide an alternative explanation of our findings.

We also acknowledge that there are many factors that are known to affect HRV (Fattison et al., 2016; Sammito and Böckelmann, 2016), such as various cardiopulmonary diseases and metabolic diseases like diabetes mellitus, as well as lifestyle habits like alcohol consumption and smoking, plus external factors like heat. Of course, even respiration itself affects HRV. It is impossible to control for every possible confound in a study, but we believe that by excluding smokers and participants who used antihypertensive or cardiological medications, we have excluded some major confounds. Our participants were also relatively young students who typically were in good physical shape. Generally speaking, it is difficult to study the relationship between HRV and metabolic demands, because the increased heart rate that accompanies intense physical exercise complicates the delineation of the different non-neural mechanisms such as respiration, which are thought to underlie HRV changes under such intense circumstances (Casadei et al., 1995, 1996; Cottin et al., 2004, 2006; Michael et al., 2017). Furthermore, chronic anxiety is associated with increased autonomic tone (for a review, see Curtis and O'Keefe, 2002), and adverse childhood events can also affect HRV (Aimie-Salleh et al., 2018; Bakema et al., 2020). This may lead to baseline differences in HRV, but the current study was not designed to address such factors. Generally speaking, we believe that additional HRV decreases as measured with our methods can be interpreted to reflect psychosocial stress; of course, there might be other, unmeasured, sources of stress as well as long-term physiological baseline differences that our methods cannot currently detect. Having said that, now that our methods to estimate reductions in additional HRV have been evaluated twice, it would be very interesting to use them to further explore the role of these kinds of factors that are known to affect HRV. In future work, we intend to combine the methods described in the current paper with between-subjects designs, which will allow us to assess baseline differences in HRV, as well as potential underlying causes of such differences.

Currently, there is debate about whether or not methods that estimate HRV, like the RMSSD method that we used in this and previous papers, should be corrected for heart rate (for a review, see de Geus et al., 2018). This is an important matter, because as the interval between two heart beats increases, so does the variability of this interval (de Geus et al.), making heart rate a potential confound. We have chosen not to correct HRV for heart rate because there is strong empirical evidence to suggest that such corrections are not necessary (e.g., Thayer et al., 2020) or even do more harm than good, by removing variance that is due to autonomic or neuropsychological processes (de Geus et al., 2018).

Our findings have clear clinical relevance: episodes of reduced additional HRV cannot be sensed by people, but they do represent periods of either psychological or physical health (or both, of course). Therefore, methods to optimally and objectively detect such episodes can be of great use to clinicians as well as to end users both by signaling epochs during which interventions are most desirable (or even required) and by teaching end users what triggers evoke such epochs. One challenge for future work will thus be to further develop ways to probe participants' reduced additional HRV periods, and to assess all possible causes of these reductions. If these are indeed strongly related to bouts of (mindless) stress and worry, probing end users (e.g., patients suffering from stress-related (psychological or somatic) pathology) on their smartphones might become a new strategy to promote learning about and dealing with stress. One could easily not only make people more aware of their physiological stress level, but also provide them with a range of interventions that can be applied in order to directly change the stress level (i.e., breathing exercises, cognitive techniques).

We are aware that the analyses reported here have been performed on relatively low numbers of participants. However, given that we have now demonstrated, in three separate datasets, that using every available data point to detect episodes of reduced additional HRV appears to be a very accurate method to do so, and given the relative computational straightforwardness of that method, we feel confident that this method is promising and will lead to accurate results. It is particularly interesting that this method requires no special calibration phase: every researcher who has HRV and movement data will be able to analyze that data 
using this method. Hopefully, further applications of this method by researchers in this field will further corroborate the validity of this method. We hope that this new method will lead to fruitful insights into reductions in additional HRV which, in turn, may lead to a better understanding of this powerful predictor of cardiovascular disease.

\section{DATA AVAILABILITY STATEMENT}

The raw data supporting the conclusions of this article will be made available by the authors, without undue reservation, to any qualified researcher.

\section{ETHICS STATEMENT}

The studies involving human participants were reviewed and approved by the Leiden University Ethics Committee. The patients/participants provided their written informed consent to participate in this study.

\section{REFERENCES}

Aimie-Salleh, N., Malarvili, M. B., and Philip, A. C. (2018). "The effect of adverse childhood experience on heart rate variability and salivary cortisol," in Proceedings of the Seventh International Conference on Advances in Computing, Electronics and Communication Kuala Lumpur.

Alwan, A. (2011). "Foreword," in Global Atlas on Cardiovascular Disease Prevention and Control (p. VI), eds S. Mendis, P. Puska, and B. Norrving (Geneva: WHO Press).

Bakema, M. J., van Zuiden, M., Collard, D., Zantvoord, J. B., de Rooij, S. R., Elsenburg, L. K., et al. (2020). Associations between child maltreatment, autonomic regulation, and adverse cardiovascular outcome in urban population: the HELIUS study. Front. Psychiatry 11:69. doi: 10.3389/fpsyt.2020.00069

Berntsen, G. G., Quigley, K. S., Norman, G. J., and Lozano, D. L. (2017). "Cardiovascular psychophysiology," in Handbook of Psychophysiology, 4th Edn, eds J. T. Cacioppo, L. G. Tassinary, and G. G. Berntson (Cambridge: Cambridge University Press), 183-216.

Blix, A. S., Stromme, S. B., and Ursin, H. (1974). Additional heart rate-An indicator of psychological activation. Aerospace Med. 45, 1219-1222.

Bosma, H., Peter, R., Siegrist, J., and Marmot, M. (1998). Two alternative job stress models and the risk of coronary heart disease. Am. J. Public Health 88, 68-74. doi: 10.2105/ajph.88.1.68

Brosschot, J. F. (2010). Markers of chronic stress: prolonged physiological activation and (un)conscious perseverative cognition. Neurosci. Biobehav. Rev. 35, 46-50. doi: 10.1016/j.neubiorev.2010.01.004

Brosschot, J. F., and Thayer, J. F. (2003). Heart rate response is longer after negative emotions than after positive emotions. Int. J. Psychophysiol. 50, 181-187. doi: 10.1016/S0167-8760(03)00146-6

Brosschot, J. F., van Dijk, E., and Thayer, J. F. (2007). Daily worry is related to low heart rate variability during waking and the subsequent nocturnal sleep period. Int. J. Psychophysiol. 63, 39-47. doi: 10.1016/j.ijpsycho.2006.07.016

Brosschot, J. F., Verkuil, B., and Thayer, J. F. (2010). Conscious and unconscious perseverative cognition: is a large part of prolonged physiological activity due to unconscious stress? J. Psychos. Res. 69, 407-416. doi: 10.1016/j.jpsychores.2010. 02.002

Brown, S. B. R. E., Brosschot, J. F., Versluis, A., Thayer, J. F., and Verkuil, B. (2018). New methods to optimally detect episodes of non-metabolic heart rate variability reduction as an indicator of psychological stress in everyday life. Int. J. Psychophysiol. 131, 30-36.

\section{AUTHOR CONTRIBUTIONS}

SB tested participants and created analysis scripts. AV tested participants. All authors contributed to the article and approved the submitted version.

\section{FUNDING}

SB was supported by a "Top"-grant of the Netherlands Organisation for Health Research and Development (ZON-MW) awarded to JB (Grant No. 40-00812-98-11029); a grant from the Den Dulk-Moermans Fonds awarded to JB (6503/21-6-16); and a research grant from the Netherlands Organization for Scientific Research (NWO Veni Grant) awarded to BV (Grant No. 4451-14-013).

\section{ACKNOWLEDGMENTS}

We would like to thank Paul Siegwardt for his assistance in testing participants.

Casadei, B., Cochrane, S., Johnston, J., Conway, J., and Sleight, P. (1995). Pitfalls in the interpretation of spectral analysis of the heart rate variability during exercise in humans. Acta Physiol. Scand. 153, 125-131. doi: 10.1111/j.1748-1716.1995. tb09843.x

Casadei, B., Moon, J., Johnston, J., Caiazza, A., and Sleight, P. (1996). Is respiratory sinus arrhythmia a good index of cardiac vagal tone in exercise? J. Appl. Physiol. 81, 556-564. doi: 10.1152/jappl.1996.81.2.556

Cottin, F., Durbin, F., and Papelier, Y. (2004). Heart rate variability during cycloergometric exercise or judo wrestling eliciting the same heart rate level. Eur. J. Appl. Physiol. 91, 177-184. doi: 10.1007/s00421-003-0969-1

Cottin, F., Lepretre, P. M., Lopes, P., Papelier, Y., Medigue, C., and Billat, V. (2006). Assessment of ventilatory thresholds from heart rate variability in well-trained subjects during cycling. Int. J. Sports Med. 27, 959-967. doi: 10.1055/s-2006923849

Curtis, B. M., and O'Keefe, J. H. Jr. (2002). Autonomic tone as a cardiovascular risk factor: the dangers of chronic fight or flight. Mayo Clin. Proc. 77, 45-54. doi: 10.4065/77.1.45

de Geus, E. J. C., Gianaros, P. J., Brindle, R. C., Jennings, J. R., and Berntson, G. G. (2018). Should heart rate variability be "corrected" for heart rate? Biological, quantitative, and interpretative considerations. Psychophysiology 56:e13287. doi: $10.1111 /$ psyp. 13287

Ebner-Priemer, U. W., Welch, S. S., Grossman, P., Reisch, T., Linehan, M. M., and Bohus, M. (2007). Psychophysiological ambulatory assessment of affective dysregulation in borderline personality disorder. Psychiatry Res. 150, 265-275. doi: 10.1016/j.psychres.2006.04.014

Fattison, J., Oswald, V., and Lalonde, F. (2016). Influence diagram of physiological and environmental factors affecting heart rate variability: an extended literature overview. Heart Int. 11, e32-e40. doi: 10.5301/heartint.5000232

Hall, M., Vasko, R., Buysse, R., Ombao, H., Chen, Q., Cashmere, J. D., Kupfer, D., and Thayer, J. F. (2004). Acute stress affects heart rate variability during sleep. Psychosom. Med. 66, 56-62. doi: 10.1097/01.psy.0000106884.58744.09

Hillebrand, S., Gast, K. B., de Mutsert, R., Swenne, C. A., Jukema, J. W., Middeldorp, S., et al. (2013). Heart rate variability and first cardiovascular event in populations without known cardiovascular disease: meta-analysis and dose-response meta-regression. Europace 15, 742-749. doi: 10.1093/europace/ eus341

Jacob, R. G., Thayer, J. F., Manuck, S. B., Muldoon, M. F., Tamres, L. K., Williams, D. M., et al. (1999). Ambulatory blood pressure responses and the circumplex model of mood: a 4-day study. Psychos. Med. 61, 319-333. doi: 10.1097/ 00006842-199905000-00011 
Jarczok, M. N., Koenig, J., Wittling, A., Fischer, J. E., and Thayer, J. F. (2019). First evaluation of an index of low vagally-mediated heart rate variability as a marker of health risks in human adults: proof of concepts. J. Clin. Med. 8:1940. doi: $10.3390 / \mathrm{jcm} 8111940$

Kivimäki, M., Virtanen, M., Elovainio, M., Kouvonen, A., Väänänen, A., and Vahtera, J. (2006). Work stress in the etiology of coronary heart disease-a meta-analysis. Scand. J. Work Environ. Health 32, 431-442.

Lazarus, R. S. (1993). From psychological stress to the emotions: a history of changing outlooks. Annu. Rev. Psychol. 44, 1-22. doi: 10.1146/annurev.ps.44. 020193.000245

Lugade, V., Fortune, E., Morrow, M., and Kaufman, K. (2014). Validity of using tri-axial accelerometers to measure human movement - part I: posture and movement detection. Med. Eng. Phys. 36, 659-669. doi: 10.1016/j.medengphy. 2014.02.006

Matthews, K. A., and Gump, B. B. (2002). Chronic work stress and marital dissolution increase risk of posttrial mortality in men from the multiple risk factor intervention trial. Arch. Intern. Med. 62, 309-315. doi: 10.1001/archinte. 162.3.309

McEwen, B. S. (2001). From molecules to mind. Stress, individual differences, and the social environment. Ann. N.Y. Acad. Sci. 935, 42-49. doi: 10.1111/j.17496632.2001.tb03469.x

Michael, S., Graham, K. S., and Davis, G. M. (2017). Cardiac autonomic responses during exercise and post-exercise recovery using heart rate variability and systolic time intervals-A review. Front. Physiol. 8:301. doi: 10.3389/fphys.2017. 00301

Myrtek, M., and Brügner, G. (1996). Perception of emotions in everyday life: studies with patients and normals. Biol. Psychol. 42, 147-165. doi: 10.1016/ 0301-0511(95)05152-x

Myrtek, M. (2004). Heart and Emotion: Ambulatory Monitoring Studies in Everyday Life. Ashland, TN: Hogrefe Publishing.

Myrtek, M., Aschenbrenner, F., and Brügner, G. (2005). Emotions in everyday life: an ambulatory monitoring study with female students. Biol. Psychol. 68, 237-255. doi: 10.1016/j.biopsycho.2004.06.001

Orth-Gomér, K., Wamala, S. P., Horsten, M., Schenck-Gustafsson, K., Schneiderman, N., and Mittleman, M. A. (2000). Marital stress worsens prognosis in women with coronary heart disease. JAMA 284, 3008-3014. doi: $10.1001 /$ jama.284.23.3008

Pieper, S., Brosschot, J. F., van der Leeden, R., and Thayer, J. (2010). Prolonged cardiac effects of momentary assessed stressful events and worry episodes. Psychos. Med. 72, 570-577. doi: 10.1097/psy.0b013e3181dbc0e9

Pieper, S., Brosschot, J. F., van der Leeden, R., and Thayer, J. F. (2007). Cardiac effects of momentary assessed worry episodes and stressful events. Psychos. Med. 69, 901-909. doi: 10.1097/psy.0b013e31815a9230

Prill, T., and Fahrenberg, J. (2007). New methods in ambulatory blood pressure monitoring: interactive monitoring and detection of posture and movement patterns. Behav. Res. Methods 39, 390-398. doi: 10.3758/BF03193008

Rennie, K. L., Hemingway, H., Kumari, M., Brunner, E., Malik, M., and Marmot, M. (2003). Effects of moderate and vigorous physical activity on heart rate variability in a British study of civil servants. Am. J. Epidemiol. 158, 135-143. doi: 10.1093/aje/kwg120

Rosengren, A., Hawken, S., Ounpuu, S., Sliwa, K., Zubaid, M., Almahmeed, W. A., et al. (2004). Association of psychosocial risk factors with risk of acute myocardial infarction in 11119 cases and 13648 controls from 52 countries (the INTERHEART study): case-control study. Lancet 364, 953-962. doi: 10.1016/ S0140-6736(04)17019-0

Sammito, S., and Böckelmann, I. (2016). Factors influencing heart rate variability. Int. Cardiovasc. Forum J. 6, 18-22. doi: 10.17987/icfj.v6i0.242

Sowder, E., Gevirtz, R. N., and Shapiro, W. (2010). Restoration of vagal tone: a possible mechanism for functional abdominal pain. Appl. Psychophysiol. Biofeedback 35, 199-206. doi: 10.1007/s10484-010-9128-8

Stewart, J. M., Montgomery, L. D., Glover, J. L., and Medow, M. S. (2007). Changes in regional blood volume and blood flow during static handgrip. Am. J. Physiol. Heart Circ. Physiol. 292, H215-H223.

Task Force of The European Society of Cardiology and The North American Society of Pacing, and Electrophysiology (1996). Heart rate variability: standards of measurement, physiological interpretation, and clinical use. Eur. Heart J. 17, 354-381. doi: 10.1093/oxfordjournals.eurheartj.a014868

Thayer, J. F., and Lane, R. D. (2007). The role of vagal function in the risk for cardiovascular disease and mortality. Biol. Psychol. 74, 224-242. doi: 10.1016/ j.biopsycho.2005.11.013

Thayer, J. F., Williams, D. P., and Tarvainen, M. P. (2020). Properties of three common time domain indices of heart rate variability: effects of detrending. Psychos. Med. 82:e1798.

Thayer, J. F., Yamamoto, S. S., and Brosschot, J. F. (2010). The relationship of heart rate variability, risk factors and cardiovascular disease. Int. J. Cardiol. 141, 122-131. doi: 10.1016/j.ijcard.2009.09.543

Verkuil, B., Brosschot, J. F., Tollenaar, M. S., Lane, R. D., and Thayer, J. F. (2016). Prolonged non-metabolic heart rate variability reduction as a physiological marker of psychological stress in daily life. Ann. Behav. Med. 50, 704-714. doi: 10.1007/S12160-016-9795-7

Versluis, A., Verkuil, B., Spinhoven, P., and Brosschot, J. F. (2018). Feasibility and effectiveness of a worry-reduction training using the smartphone: a pilot randomized controlled trial. Br. J. Guid. Counsel. 48, 227-239. doi: 10.1080/ 03069885.2017.1421310

Conflict of Interest: The authors declare that the research was conducted in the absence of any commercial or financial relationships that could be construed as a potential conflict of interest.

Copyright (c) 2020 Brown, Brosschot, Versluis, Thayer and Verkuil. This is an openaccess article distributed under the terms of the Creative Commons Attribution License (CC BY). The use, distribution or reproduction in other forums is permitted, provided the original author(s) and the copyright owner(s) are credited and that the original publication in this journal is cited, in accordance with accepted academic practice. No use, distribution or reproduction is permitted which does not comply with these terms. 